\title{
Quantum light-induced nonadiabatic phenomena in the absorption spectrum of formaldehyde: Full- and reduced-dimensionality studies
}

\author{
Cite as: J. Chem. Phys. 153, 234302 (2020); doi: 10.1063/5.0035870 \\ Submitted: 31 October 2020 - Accepted: 30 November 2020 • \\ Published Online: 17 December 2020
}

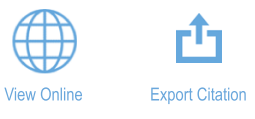

Csaba Fábri, ${ }^{1,2, a)}$ (D) Benjamin Lasorne, ${ }^{3}$ (D) Gábor J. Halász,, (D) Lorenz S. Cederbaum, ${ }^{5}$ (D) and Ágnes Vibók ${ }^{6,7, b)}$ (D)

\begin{abstract}
AFFILIATIONS
'Laboratory of Molecular Structure and Dynamics, Institute of Chemistry, Eötvös Loránd University, Pázmány Péter Sétány 1/A, H-1117 Budapest, Hungary

${ }_{2}^{2}$ MTA-ELTE Complex Chemical Systems Research Group, P.O. Box 32, H-1518 Budapest 112, Hungary

${ }^{3}$ Institut Charles Gerhardt Montpellier (ICGM), Université de Montpellier, CNRS, ENSCM, F-34095 Montpellier, France

${ }^{4}$ Department of Information Technology, University of Debrecen, P.O. Box 400, H-4002 Debrecen, Hungary

${ }^{5}$ Theoretische Chemie, Physikalisch-Chemisches Institut, Universität Heidelberg,

Im Neuenheimer Feld 229, 69120 Heidelberg, Germany

${ }^{6}$ Department of Theoretical Physics, University of Debrecen, P.O. Box 400, H-4002 Debrecen, Hungary

${ }^{7}$ ELI-ALPS, ELI-HU Non-Profit Ltd., Dugonics tér 13, H-6720 Szeged, Hungary
\end{abstract}

Note: This paper is part of the JCP Special Topic on Polariton Chemistry: Molecules in Cavities and Plasmonic Media.

a) Electronic mail: ficsaba@caesar.elte.hu

b) Author to whom correspondence should be addressed: vibok@phys.unideb.hu

\begin{abstract}
The coupling of a molecule to a cavity can induce conical intersections of the arising polaritonic potential energy surfaces. Such intersections give rise to the strongest possible nonadiabatic effects. By choosing an example that does not possess nonadiabatic effects in the absence of the cavity, we can study, for the first time, the emergence of these effects in a polyatomic molecule due to its coupling with the cavity taking into account all vibrational degrees of freedom. The results are compared with those of reduced-dimensionality models, and the shortcomings and merits of the latter are analyzed.
\end{abstract}

Published under license by AIP Publishing. https://doi.org/10.1063/5.0035870

\section{INTRODUCTION}

Conical intersections (CIs) are degeneracy points of adiabatic potential energy surfaces (PESs) in the multidimensional vibrational configuration space of polyatomic molecules. In this case, nonadiabaticity couples the participating electronic states, and the nonadiabatic coupling becomes extremely large or even singular at the close vicinity of CIs, leading to the extreme breakdown of the Born-Oppenheimer approximation (BOA). ${ }^{1-6}$ It is now widely accepted that CIs play a key mechanistic role in photophysics, photochemistry, and photobiology ${ }^{7-19}$ as they give rise to exceptionally fast radiationless transitions, typically on the femtosecond time scale.
Generally, nonadiabatic phenomena are intrinsic properties of molecular systems owing to the strong coupling between the nuclei and electrons, but nonadiabatic effects can also be induced either by classical laser light ${ }^{20,21}$ or by quantum light present in a cavity. ${ }^{22,23}$ In both cases, the light couples different degrees of freedom (dofs) of the molecule, and a so-called light-induced conical intersection (LICI) arises. In contrast to natural CIs in field-free polyatomic molecules, the energetic and spatial position of LICIs can be controlled by varying the parameters of the laser field or cavity. The former case (classical laser light) has already been discussed several times, mainly in diatomics, ${ }^{24-27}$ but in some situations in polyatomic molecules ${ }^{28-30}$ as well, both theoretically ${ }^{24-30}$ and experimentally. ${ }^{31-33}$ However, the literature on the merits of the latter area 
(quantum light) is still rather incomplete. This is due to the fact that polaritonic chemistry has recently become an emerging and one of the most promising fields of physical chemistry. ${ }^{22}$

The interaction between a molecule and a cavity mode, depending on the frequency of the cavity mode, can couple either two or more electronic states of the molecule (optical cavity) or vibrational states (infrared cavity) on a single PES. In the former case, the socalled vibronic polaritons emerge, while the latter situation leads to the formation of vibrational polaritons. Owing to the possibility of manipulating the reactivity of molecules inside infrared cavities, the field of vibrational strong coupling is a hot topic area. ${ }^{59-76}$

In the present work, however, our aim is to study the effect of strong resonant coupling between different electronic states in the optical frequency range, leading to the formation of vibronic polaritons. We demonstrate that in a molecule with several vibrational dofs, the coupling between the photonic, vibrational, and electronic dofs gives rise to the so-called quantum LICI. ${ }^{77}$ Our system of interest is the four-atomic $\mathrm{H}_{2} \mathrm{CO}$ (formaldehyde) molecule, which is described with full-dimensional (6D) as well as reduced two- and one-dimensional (2D and 1D) quantum-dynamical models within the cavity-quantum-electrodynamical (cQED) framework. This molecule is a suitable candidate to investigate quantum-lightinduced nonadiabatic phenomena as it does not possess any inherent nonadiabatic effects in the studied region of the nuclear configuration space. ${ }^{78-80}$ Therefore, nonadiabatic effects that appear in the cavity can be clearly attributed to the quantum LICI that is shown in Fig. 1 along with the three lowest polaritonic surfaces of $\mathrm{H}_{2} \mathrm{CO}$ coupled to a cavity mode.

First, the dressed absorption spectrum of the molecule in the cavity is computed by an accurate all-mode quantum-dynamical procedure, and results are compared to the absorption spectrum of the field-free molecule. Then, we study the dressed absorption spectrum using reduced-dimensional quantum-dynamical models; specifically, 2D and 1D computations are performed for a wide range of the cavity coupling strength parameter. It will be demonstrated that while the $6 \mathrm{D}$ and $2 \mathrm{D}$ results exhibit a qualitative resemblance, the $1 \mathrm{D}$ model is unable to provide a description of the field-free or dressed absorption spectrum of the molecule. At least a 2D approximation is needed to properly describe the quantum-light-induced nonadiabatic effects.

While all-mode dynamics of polyatomic molecules has been, by now, amply investigated in natural CI situations, ${ }^{81,82}$ this is not the case for polyatomic molecules in the cavity. Here, the present study is the first example.

This paper is organized as follows. In Sec. II, the working Hamiltonian and the quantum-dynamical description of a molecule coupled to a cavity mode are introduced. Section III is devoted to

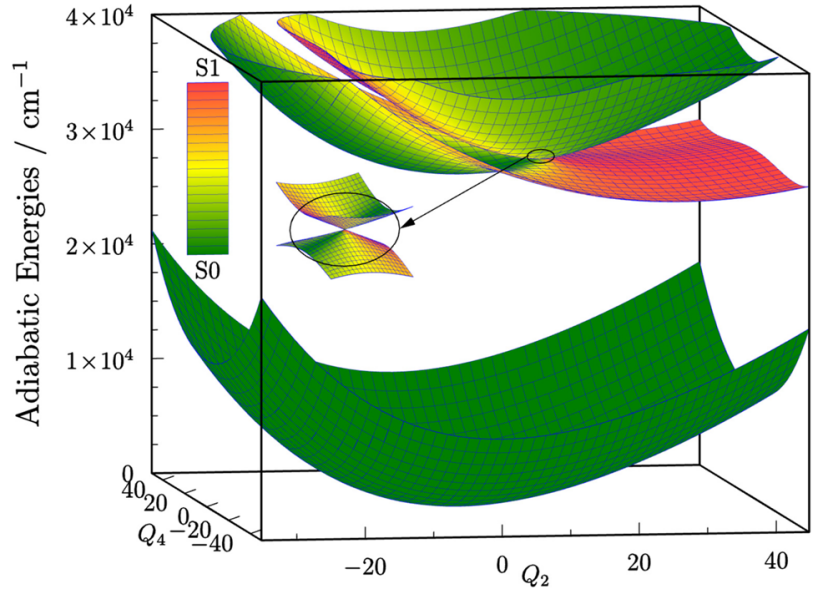

FIG. 1. Three lowest polaritonic (adiabatic) surfaces of $\mathrm{H}_{2} \mathrm{CO}$ coupled to a single cavity mode. $Q_{2}$ and $Q_{4}$ are the normal coordinates of the $v_{2}(C=0$ stretch) and $v_{4}$ (out-of-plane bend) vibrational modes. The cavity wavenumber and coupling strength are chosen as $\omega_{\mathrm{c}}=29000 \mathrm{~cm}^{-1}$ and $g=5.97 \times 10^{-2}$ a.u., respectively. The light-induced conical intersection between the second and third polaritonic surfaces is shown in the inset. The character of the polaritonic surfaces is indicated by different colors (see the legend on the left; the labels $S_{0}$ and $S_{1}$ refer to the ground and excited electronic states of the molecule).

the discussion of our results and is divided into three subsections. In Subsection III A, the 6D field-free and dressed spectra are presented and compared. In Subsection III B, the field-free and dressed spectra obtained with a 2D quantum-dynamical model are analyzed. In Subsection III C, the performance of a $1 \mathrm{D}$ quantum-dynamical model is discussed. The conclusions are summarized in Sec. IV.

\section{THEORY AND COMPUTATIONAL PROTOCOL}

The Hamiltonian of a molecule coupled to a single cavity mode $^{83}$ takes the form

$$
\hat{H}=\hat{H}_{0}+\hbar \omega_{\mathrm{c}} \hat{a}^{\dagger} \hat{a}-g \hat{\vec{\mu}} \vec{e}\left(\hat{a}^{\dagger}+\hat{a}\right),
$$

where $\hat{H}_{0}$ corresponds to the Hamiltonian of the field-free molecule, $\omega_{\mathrm{c}}$ is the angular frequency of the cavity mode, $\hat{a}^{\dagger}$ and $\hat{a}$ are creation and annihilation operators associated with the cavity mode, $g$ is the coupling strength parameter, $\hat{\vec{\mu}}$ denotes the electric dipole moment operator, and $\vec{e}$ is the polarization vector. If two electronic states are taken into account, the Hamiltonian can be written as

$$
\hat{H}=\left[\begin{array}{ccccccc}
\hat{T}+V_{\mathrm{X}} & 0 & W_{1}^{\mathrm{X}} & W_{1} & 0 & 0 & \cdots \\
0 & \hat{T}+V_{\mathrm{A}} & W_{1} & W_{1}^{\mathrm{A}} & 0 & 0 & \cdots \\
W_{1}^{\mathrm{X}} & W_{1} & \hat{T}+V_{\mathrm{X}}+\hbar \omega_{\mathrm{c}} & 0 & W_{2}^{\mathrm{X}} & W_{2} & \cdots \\
W_{1} & W_{1}^{\mathrm{A}} & 0 & \hat{T}+V_{\mathrm{A}}+\hbar \omega_{\mathrm{c}} & W_{2} & W_{2}^{\mathrm{A}} & \cdots \\
0 & 0 & W_{2}^{\mathrm{X}} & W_{2} & \hat{T}+V_{\mathrm{X}}+2 \hbar \omega_{\mathrm{c}} & 0 & \cdots \\
0 & 0 & W_{2} & W_{2}^{\mathrm{A}} & 0 & \hat{T}+V_{\mathrm{A}}+2 \hbar \omega_{\mathrm{c}} & \cdots \\
\vdots & \vdots & \vdots & \vdots & \vdots & \vdots & \ddots
\end{array}\right],
$$


where $\hat{T}$ is the kinetic energy operator, $V_{\mathrm{X}}$ and $V_{\mathrm{A}}$ denote the ground-state and excited-state PESs, $W_{n}=-g \sqrt{n} \vec{d} \vec{e}$, with $\vec{d}$ being the transition dipole moment (TDM) vector, and $W_{n}^{\mathrm{X}}=-g \sqrt{n} \vec{d} \times \vec{e}$ and $W_{n}^{\mathrm{A}}=-g \sqrt{n} \vec{d}_{\mathrm{A}} \vec{e}$, where $\vec{d}_{\mathrm{x}}$ and $\vec{d}_{\mathrm{A}}$ refer to the permanent dipole moments (PDMs) associated with the electronic states X and A, respectively. The polaritonic (adiabatic) PESs (see also Fig. 1) can be obtained as eigenvalues of the potential energy part of the Hamiltonian of Eq. (2) at each nuclear configuration.

The eigenstates of the Hamiltonian of Eq. (2),

$$
\left|\Phi_{k}\right\rangle=\sum_{\alpha=\mathrm{X}, \mathrm{A}} \sum_{i} \sum_{n} c_{\alpha i n}^{(k)}|\alpha i\rangle|n\rangle
$$

can be written as the linear combination of the products of fieldfree molecular vibronic eigenstates (denoted by $|\mathrm{X} i\rangle$ and $|\mathrm{A} i\rangle$ ) and Fock states $|n\rangle(n=0,1,2, \ldots)$ of the cavity mode. The intensities of electric dipole transitions between the eigenstates $\left|\Phi_{k}\right\rangle$ and $\left|\Phi_{l}\right\rangle$ can be obtained as

$$
I_{k l} \propto \omega_{k l} \sum_{\alpha=x, y, z}\left|\left\langle\Phi_{k}\left|\hat{\mu}_{\alpha}\right| \Phi_{l}\right\rangle\right|^{2},
$$

where $\omega_{k l}$ is the angular frequency of the transition $\left|\Phi_{k}\right\rangle \rightarrow\left|\Phi_{l}\right\rangle$ and $\hat{\mu}_{\alpha}$ denotes the components of the electric dipole moment operator.

The planar equilibrium structure of $\mathrm{H}_{2} \mathrm{CO}\left(C_{2 v}\right.$ point-group symmetry) in its electronic ground state $(\mathrm{X})$ is shown in Fig. 2, while the normal modes of $\mathrm{H}_{2} \mathrm{CO}$ are summarized in Table I. The definition of the Cartesian axes in Fig. 2 corresponds to the Mulliken convention. ${ }^{84}$ The excited electronic state (A) has a double-well structure along the $v_{4}$ (out-of-plane) vibrational mode, and the two equivalent nonplanar equilibrium structures are connected by a planar transition state structure. The $V_{\mathrm{X}}\left(\mathrm{S}_{0}\right.$ electronic state, $\left.\mathrm{X}\right)$ and $V_{\mathrm{A}}\left(\mathrm{S}_{1}\right.$ electronic state, A) PESs were taken from Refs. 85 and 86 , respectively.

The six-dimensional (6D) vibrational Schrödinger equation was solved variationally by the numerically exact and general rovibrational program system GENIUSH ${ }^{87-89}$ for both electronic states. The body-fixed Cartesian position vectors of the nuclei were parameterized using polyspherical coordinates, ${ }^{90}$ and the body-fixed axes were oriented according to the Eckart conditions ${ }^{91}$ using the equilibrium structure of the $\mathrm{X}$ electronic state as the reference structure.

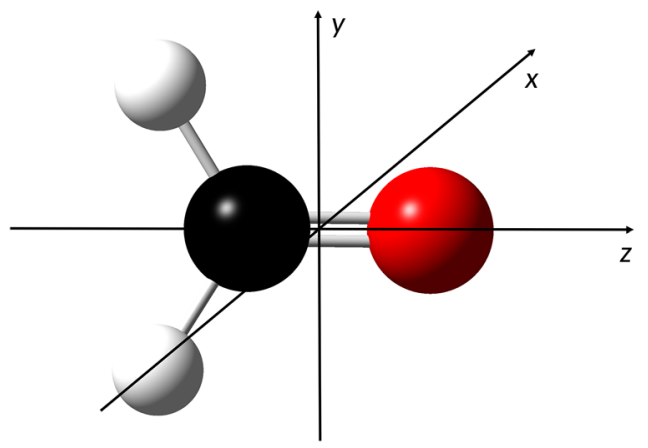

FIG. 2. Equilibrium structure of the $\mathrm{H}_{2} \mathrm{CO}$ molecule in its electronic ground state $(X)$ and the body-fixed coordinate system chosen (the molecule is placed in the $y z$ plane).
TABLE I. Normal mode labels, $C_{2} v$ irreducible representations, description of normal modes, and anharmonic fundamentals $\left[\omega_{X}\right.$ and $\omega_{A}$, obtained by six-dimensional variational computations in the electronic ground $(X)$ and excited $(A)$ states of $\mathrm{H}_{2} \mathrm{CO}$, in units of $\mathrm{cm}^{-1}$ ].

\begin{tabular}{ccccc}
\hline \hline Mode & Symmetry & Description & $\omega_{\mathrm{X}} / \mathrm{cm}^{-1}$ & $\omega_{\mathrm{A}} / \mathrm{cm}^{-1}$ \\
\hline$v_{1}$ & $\mathrm{~A}_{1}$ & Sym C-H stretch & 2728.4 & 2842.3 \\
$v_{2}$ & $\mathrm{~A}_{1}$ & $\mathrm{C}=\mathrm{O}$ stretch & 1738.1 & 1181.9 \\
$v_{3}$ & $\mathrm{~A}_{1}$ & $\mathrm{CH}_{2}$ scissor & 1466.0 & 1309.5 \\
$v_{4}$ & $\mathrm{~B}_{1}$ & Out-of-plane bend & 1147.0 & 137.0 \\
$v_{5}$ & $\mathrm{~B}_{2}$ & Antisym C-H stretch & 2819.9 & 2965.4 \\
$v_{6}$ & $\mathrm{~B}_{2}$ & $\mathrm{CH}_{2}$ rock & 1234.5 & 877.9 \\
\hline \hline
\end{tabular}

The rotational dofs were omitted from our computational protocol, and the molecule was fixed with respect to the external electric field. A symmetry-adapted 6D direct-product discrete variable representation (DVR) basis and atomic mass values $\left(m_{\mathrm{C}}=12.0 \mathrm{u}\right.$, $m_{\mathrm{O}}=15.994915 \mathrm{u}$, and $m_{\mathrm{H}}=1.007825 \mathrm{u}$ ) were employed throughout the nuclear motion computations. The $S_{2}^{*}$ molecular symmetry group ${ }^{92}$ that is isomorphic to the $C_{2 v}$ point group was fully exploited using the method reported in Ref. 93, and the vibrational Hamiltonian matrix was separated into four noninteracting blocks corresponding to the four irreducible representations of the $S_{2}^{*}$ molecular symmetry group. Vibrational quantum numbers were assigned to the vibrational eigenstates by counting the nodes of one-dimensional wave function cuts along the different normal coordinates.

The permanent and transition dipole moment, i.e., PDM and TDM, respectively, surfaces required by the computation of the dressed states and transition amplitudes were generated by a second-order Taylor expansion using the polyspherical coordinates described above. The Taylor series were centered at the equilibrium structure of the X electronic state (TDM and X-state PDM) and at the planar transition state structure of the A electronic state (A-state PDM), respectively. The PDM and TDM components were referenced in the Eckart frame used by the vibrational eigenstate computations, and dipole derivatives were evaluated by the finite-difference method at the CAM-B3LYP/6-31G* level of theory. The symmetry properties of the body-fixed PDM and TDM components are summarized in Table II. Note that all components of the TDM vanish at geometries of the $C_{2 v}$ symmetry. The $6 \mathrm{D}$ dressed states were computed by diagonalizing the Hamiltonian of Eq. (2) in the directproduct basis of field-free molecular eigenstates and Fock states of the cavity mode with $n=0,1,2$.

TABLE II. Symmetry properties ( $C_{2 v}$ irreducible representations) of the body-fixed components of the permanent (PDM) and transition (TDM) dipole moments.

\begin{tabular}{ccc}
\hline \hline Component & PDM & TDM \\
\hline$x$ & $\mathrm{~B}_{1}$ & $\mathrm{~B}_{2}$ \\
$y$ & $\mathrm{~B}_{2}$ & $\mathrm{~B}_{1}$ \\
$z$ & $\mathrm{~A}_{1}$ & $\mathrm{~A}_{2}$ \\
\hline \hline
\end{tabular}


In addition to the $6 \mathrm{D}$ model, two reduced-dimensional quantum-dynamical models were employed. The $2 \mathrm{D}\left(v_{2}, v_{4}\right)$ and $1 \mathrm{D}\left(v_{4}\right)$ models were defined by evaluating the normal coordinates at the planar transition state structure of the A electronic state. The inactive normal coordinates were set to zero, and the $2 \mathrm{D}\left(v_{2}, v_{4}\right)$ and $1 \mathrm{D}\left(v_{4}\right)$ PESs $\left(V_{\mathrm{X}}\right.$ and $\left.V_{\mathrm{A}}\right)$ and TDM surfaces were calculated at the CAM-B3LYP/6-31G* level of theory. The energy levels and eigenstates of the coupled molecule-cavity system were computed by diagonalizing the Hamiltonian of Eq. (2) in the direct-product basis of $2 \mathrm{D}$ or $1 \mathrm{D}$ DVR basis functions and Fock states of the cavity mode with $n=0,1,2,3$.

In the $2 \mathrm{D}\left(v_{2}, v_{4}\right)$ model, the branching plane is spanned by the vibrational modes $v_{2}$ ( $\mathrm{A}_{1}$ symmetry) and $v_{4}$ ( $\mathrm{B}_{1}$ symmetry) such that $v_{2}$ is the tuning mode and $v_{4}$ is the coupling mode. As only the bodyfixed $y$ component of the TDM can be nonzero in the $2 \mathrm{D}\left(v_{2}, v_{4}\right)$ case, the electric field must have a nonzero component along the $y$ axis. This gives rise to a LICI (see Fig. 1) and possible geometric phase effects around the LICI. The dressing allows the two electronic states of different symmetries $\left(\mathrm{A}_{1}\right.$ and $\left.\mathrm{A}_{2}\right)$ to cross along $v_{2}$ (preserving $C_{2 v}$ symmetry), and the light-matter coupling between the two electronic states can linearly increase from zero $\left(C_{2 v}\right.$ symmetry) along $v_{4}$. In the $6 \mathrm{D}$ case, we can also consider the body-fixed $x$ component of the TDM such that the actual coupling mode will be a linear combination of the modes $v_{4}\left(\mathrm{~B}_{1}\right), v_{5}\left(\mathrm{~B}_{2}\right)$, and $v_{6}\left(\mathrm{~B}_{2}\right)$ determined by the angle between the electric field and the TDM in the $x y$ plane. The effective tuning mode will be a linear combination of different modes with contributions from all three $\mathrm{A}_{1}$ vibrational modes $v_{1}$, $v_{2}$, and $v_{3}$, dominated by $v_{2}$. Group theoretical considerations imply that the first-order nonadiabatic coupling is zero at $C_{2 v}$ geometries (such as the Franck-Condon point) and small otherwise at neighboring $C_{s}$ geometries with distortions along a vibrational mode of $\mathrm{B}_{1}$ or $\mathrm{B}_{2}$ symmetry.

\section{RESULTS AND DISCUSSIONS}

Having described the theory and the molecular system under investigation, the absorption spectrum of $\mathrm{H}_{2} \mathrm{CO}$, either field free (i.e., no cavity) or dressed (coupled to a cavity mode), is discussed. We focus on the spectrum consisting of vibronic transitions from

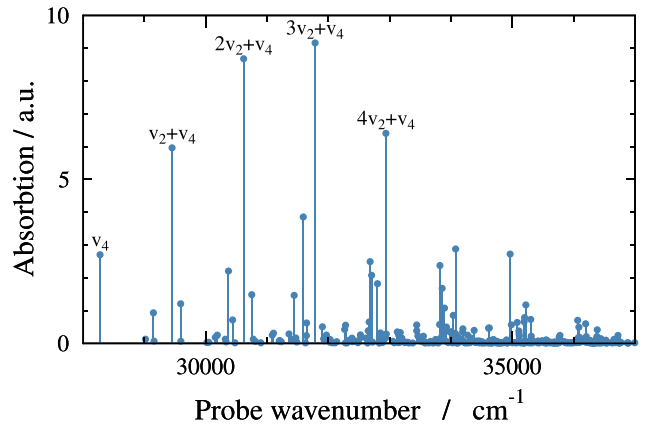

FIG. 3. Absorption spectrum of the field-free $\mathrm{H}_{2} \mathrm{CO}$ molecule obtained with the $6 \mathrm{D}$ quantum-dynamical model. Peaks that are members of the $v_{4}$ (out-of-plane bend) progression with different numbers of quanta in the $v_{2}(\mathrm{C}=\mathrm{O}$ stretch) vibrational mode are labeled $n v_{2}+v_{4}$, where $n=0, \ldots, 4$. the electronic state $\mathrm{X}$ to A. Besides the full-dimensional, i.e., $6 \mathrm{D}$, Hamiltonian, two reduced-dimensional models, treating the $v_{2}$ and $v_{4}$ vibrational modes [2D $\left(v_{2}, v_{4}\right)$ model] or the $v_{4}$ vibrational mode $\left[1 \mathrm{D}\left(v_{4}\right)\right.$ model], are employed and their results are compared with each other. For all models considered, the initial state of the spectral transitions is chosen as either the vibrational ground state of the $\mathrm{X}$ state, $|\mathrm{X}, 0\rangle$ (field-free case), or the lowest-energy dressed state (dressed case). For all cavity parameters investigated here, the lowest-energy dressed state equals $|\mathrm{X}, 0\rangle|0\rangle$ (product of $|\mathrm{X}, 0\rangle$ and the vacuum state $|0\rangle$ of the cavity mode) to a good approximation. The final states of the spectral transitions can be approximately assigned to the singly occupied subspace, i.e., to a mixture of the

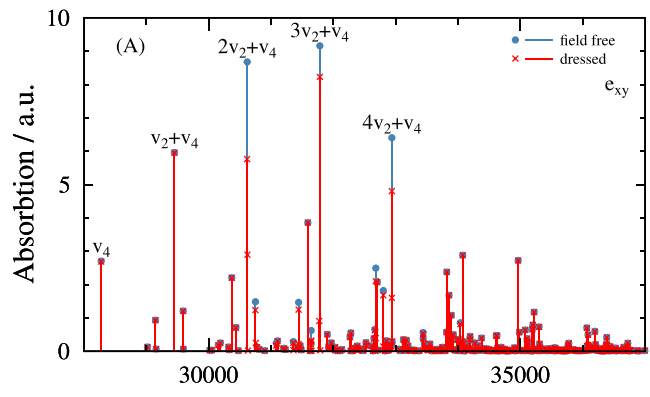

Probe wavenumber / $\mathrm{cm}^{-1}$

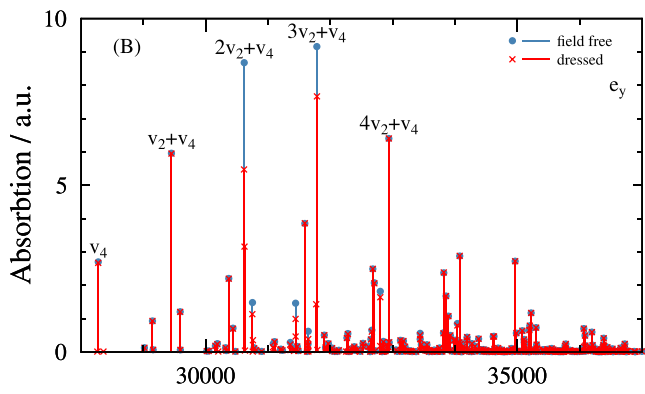

Probe wavenumber / $\mathrm{cm}^{-1}$

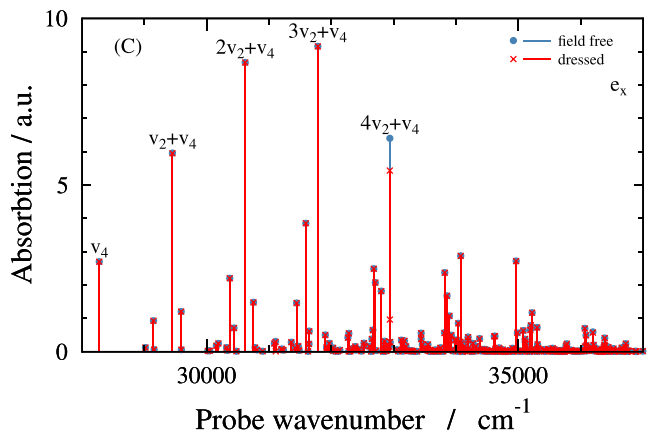

FIG. 4. Field-dressed (red) and field-free (blue) spectra of the $\mathrm{H}_{2} \mathrm{CO}$ molecule obtained with the $6 \mathrm{D}$ quantum-dynamical model. The cavity wavenumber and coupling strength values equal $\omega_{\mathrm{c}}=23794.0 \mathrm{~cm}^{-1}$ and $g=8.44 \times 10^{-4}$ a.u. Panels (A)-(C) show dressed spectra with polarization vectors $\mathbf{e}_{x y}=(1,1,0) / \sqrt{2}, \mathbf{e}_{y}=$ $(0,1,0)$, and $\mathbf{e}_{x}=(1,0,0)$, respectively. Peaks of the field-free spectrum in the $v_{4}$ (out-of-plane bend) progression with different numbers of quanta in the $v_{2}(\mathrm{C}=0$ stretch) vibrational mode are labeled $n v_{2}+v_{4}$, where $n=0, \ldots, 4$. 
molecule in its ground electronic state with one cavity photon and the molecule in the excited electronic state with zero photon.

\section{A. Full-dimensional results}

Figure 3 presents the field-free spectrum of $\mathrm{H}_{2} \mathrm{CO}$ obtained with the $6 \mathrm{D}$ quantum-dynamical model. The $6 \mathrm{D}$ field-free absorption spectrum contains several groups of peaks that correspond to transitions from the $\mathrm{X}$ vibrational ground state to the A vibrational states. Since nonadiabatic couplings are negligible in the absence of an external field, the transitions can be labeled by pure vibrational

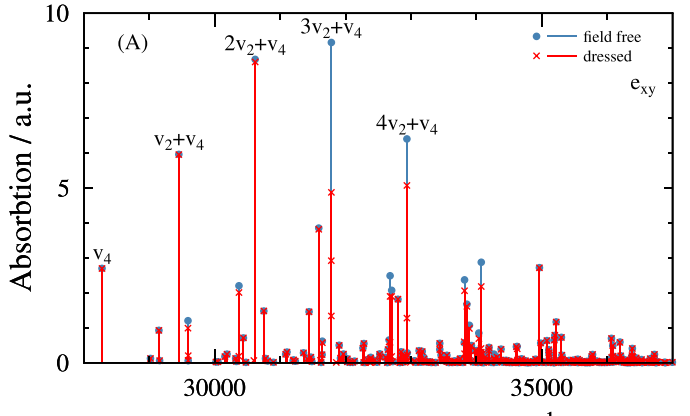

Probe wavenumber $/ \mathrm{cm}^{-1}$

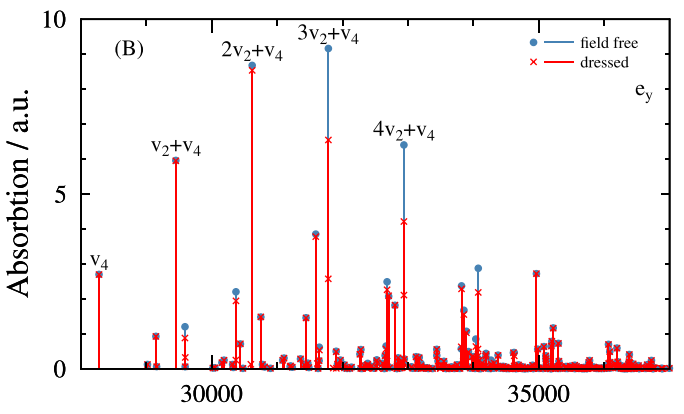

Probe wavenumber $/ \mathrm{cm}^{-1}$

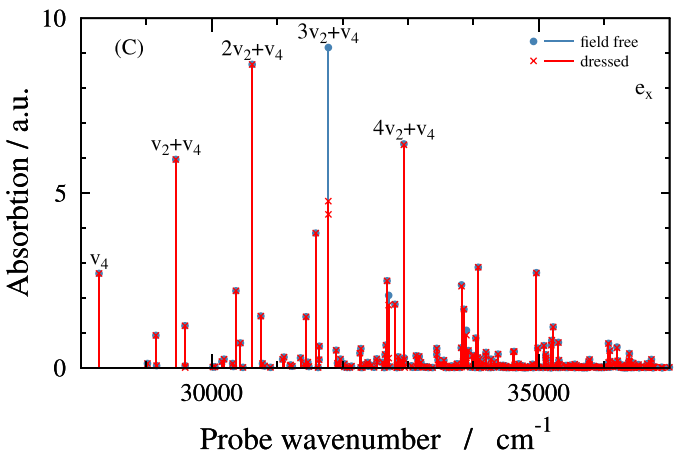

FIG. 5. Field-dressed (red) and field-free (blue) spectra of the $\mathrm{H}_{2} \mathrm{CO}$ molecule obtained with the $6 \mathrm{D}$ quantum-dynamical model. The cavity wavenumber and coupling strength values equal $\omega_{\mathrm{c}}=25575.0 \mathrm{~cm}^{-1}$ and $g=8.44 \times 10^{-4}$ a.u. Panels $(A)-(C)$ show dressed spectra with polarization vectors $\mathbf{e}_{x y}=(1,1,0) / \sqrt{2}$, $\mathbf{e}_{y}=(0,1,0)$, and $\mathbf{e}_{x}=(1,0,0)$, respectively. Peaks of the field-free spectrum in the $v_{4}$ (out-of-plane bend) progression with different numbers of quanta in the $v_{2}$ $\left(\mathrm{C}=0\right.$ stretch) vibrational mode are labeled $n v_{2}+v_{4}$, where $n=0, \ldots, 4$. quantum numbers. High-intensity peaks that belong to the $v_{4}$ progression are explicitly labeled in Fig. 3 with the quantum numbers $n v_{2}+v_{4}$ of the final A vibrational states. Note that a similar progression can be observed in the absorption spectrum from the $\mathrm{X}$ vibrational ground state to the A vibrational states $\left|\mathrm{A}, n v_{2}+3 v_{4}\right\rangle$. The $6 \mathrm{D}$ field-free spectrum in Fig. 3 shows good agreement with earlier experimental and computational results. ${ }^{94,95}$

The $6 \mathrm{D}$ spectra of $\mathrm{H}_{2} \mathrm{CO}$ coupled to a cavity mode are depicted in Figs. 4 and 5. For brevity, we call such spectra dressed spectra. The wavenumbers of the cavity mode were chosen as $\omega_{\mathrm{c}}=23794.0 \mathrm{~cm}^{-1}$ (Fig. 4) and $\omega_{\mathrm{c}}=25575.0 \mathrm{~cm}^{-1}$ (Fig. 5), both with a coupling strength value of $g=8.44 \times 10^{-4}$ a.u., corresponding to a classical field intensity of $I=10^{11} \mathrm{~W} / \mathrm{cm}^{2}$. In order to examine the effect of the polarization vector of the electric field on the dressed spectra, we have applied different polarization vectors, all referenced in the body-fixed frame, for both cavity wavenumber values. As the bodyfixed $z$ component of the TDM transforms according to the $\mathrm{A}_{2}$ irreducible representation (see Table II) and the molecule does not have any vibrational modes of $\mathrm{A}_{2}$ symmetry, the Taylor-expansion of the TDM $z$ component does not involve any first-order coupling terms for electric fields polarized along $z$, and the impact of this component is expected to be weak. We assume that the electric field is linearly polarized and choose the polarization vectors $\mathbf{e}_{x y}=(1,1,0) / \sqrt{2}$, $\mathbf{e}_{x}=(1,0,0)$, and $\mathbf{e}_{y}=(0,1,0)$.

The 6D dressed spectra in Figs. 4 and 5 show splittings of certain peaks of the 6D field-free spectrum. The cavity wavenumbers $\omega_{\mathrm{c}}=23794.0 \mathrm{~cm}^{-1}$ and $\omega_{\mathrm{c}}=25575.0 \mathrm{~cm}^{-1}$ were chosen such that more peaks of the field-free $v_{4}$ progression are split due to the interaction of the molecule with the cavity mode. It is striking in Figs. 4 and 5 that the $6 \mathrm{D}$ dressed spectra are sensitive to the choice of the polarization vector. In the following, the mechanism of the peak splittings and the polarization dependence of the $6 \mathrm{D}$ dressed spectra will be elucidated.

If the cavity wavenumber is chosen as $\omega_{\mathrm{c}}=23794.0 \mathrm{~cm}^{-1}$, the field-free peaks $2 v_{2}+v_{4}, 3 v_{2}+v_{4}$, and $4 v_{2}+v_{4}$ (besides others) are split by the coupling between the molecule and the cavity mode. The origin of these splittings can be understood by examining those eigenstates of the coupled cavity-molecule system to which the transitions occur from the initially populated lowest-energy dressed

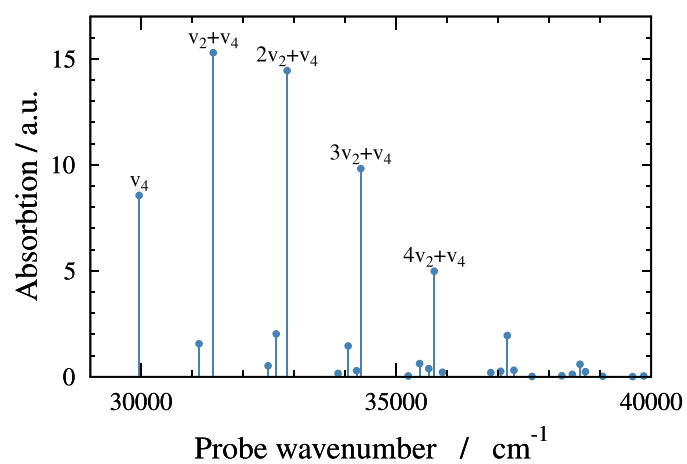

FIG. 6. Absorption spectrum of the field-free $\mathrm{H}_{2} \mathrm{CO}$ molecule obtained with the $2 \mathrm{D}\left(v_{2}, v_{4}\right)$ quantum-dynamical model. Peaks that are members of the $v_{4}$ (out-ofplane bend) progression with different numbers of quanta in the $v_{2}(\mathrm{C}=\mathrm{O}$ stretch) vibrational mode are labeled $n v_{2}+v_{4}$, where $n=0, \ldots, 4$. 
state. The cavity wavenumber of $\omega_{c}=23794.0 \mathrm{~cm}^{-1}$ is nearly resonant with the transitions $\left|\mathrm{X}, 6 v_{4}\right\rangle\left(6824.6 \mathrm{~cm}^{-1}, \mathrm{~A}_{1}\right.$ symmetry) $\rightarrow\left|\mathrm{A}, 2 v_{2}+v_{4}\right\rangle\left(30617.1 \mathrm{~cm}^{-1}, \mathrm{~B}_{1}\right)\left(\mathrm{t}_{1}\right),\left|\mathrm{X}, 2 v_{2}+4 v_{4}\right\rangle\left(7979.9 \mathrm{~cm}^{-1}\right.$, $\left.\mathrm{A}_{1}\right) \rightarrow\left|\mathrm{A}, 3 v_{2}+v_{4}\right\rangle\left(31782.2 \mathrm{~cm}^{-1}, \mathrm{~B}_{1}\right)\left(\mathrm{t}_{2}\right)$, and $\left|\mathrm{X}, 3 v_{3}+3 v_{4}+v_{6}\right\rangle$ $\left(9143.4 \mathrm{~cm}^{-1}, \mathrm{~A}_{2}\right) \rightarrow\left|\mathrm{A}, 4 v_{2}+v_{4}\right\rangle\left(32937.2 \mathrm{~cm}^{-1}, \mathrm{~B}_{1}\right)\left(\mathrm{t}_{3}\right)$. Due to the field, dressed states are formed, all of which are approximately of the form $c_{\mathrm{X} i 1}|\mathrm{X} i\rangle|1\rangle+c_{\mathrm{A} j 0}|\mathrm{~A} j\rangle|0\rangle$, where $|\mathrm{X} i\rangle$ and $|\mathrm{A} j\rangle$ denote eigenstate pairs of $t_{1}, t_{2}$, or $t_{3}$. For the state pairs of $t_{1}$ and $t_{2}$, the interacting $\mathrm{X}$ and $\mathrm{A}$ vibrational states are of $\mathrm{A}_{1}$ and $\mathrm{B}_{1}$ symmetries, respectively. Therefore, according to Table II, the $\mathrm{X}$ and A vibrational states can be coupled by the $y$ component of the TDM ( $B_{1}$ symmetry) in these two cases. On the other hand, the $\mathrm{X}$ and $\mathrm{A}$ vibrational states of $\mathrm{t}_{3}$ are of $A_{2}$ and $B_{1}$ symmetries, respectively, implying that these states can be coupled by the $x$ component of the TDM ( $\mathrm{B}_{2}$ symmetry). In view of these observations, the polarization dependence of the $6 \mathrm{D}$ dressed spectra in Fig. 4 can be readily interpreted. If the polarization vector equals $\mathbf{e}=\mathbf{e}_{x y}$, both the $\mathrm{A}_{1} \leftrightarrow \mathrm{B}_{1}$ and $\mathrm{A}_{2} \leftrightarrow \mathrm{B}_{1}$ couplings are allowed, and the field-free peaks $2 v_{2}+v_{4}, 3 v_{2}+v_{4}$, and $4 v_{2}+v_{4}$ are all split into two peaks in the $6 \mathrm{D}$ dressed spectrum. Similarly, if $\mathbf{e}=\mathbf{e}_{y}$ or $\mathbf{e}=\mathbf{e}_{x}$, the peaks $2 v_{2}+v_{4}$ and $3 v_{2}+v_{4}$ or $4 v_{2}+v_{4}$ are split, respectively.

If $\omega_{\mathrm{c}}$ is set to $25575.0 \mathrm{~cm}^{-1}$ (see Fig. 5), the transitions $\left|\mathrm{X}, v_{3}+2 v_{4}+2 v_{6}\right\rangle\left(6206.5 \mathrm{~cm}^{-1}, \mathrm{~A}_{1}\right) \rightarrow\left|\mathrm{A}, 3 v_{2}+v_{4}\right\rangle$ $\left(31782.2 \mathrm{~cm}^{-1}, \mathrm{~B}_{1}\right),\left|\mathrm{X}, 3 v_{4}+v_{5}\right\rangle\left(6207.1 \mathrm{~cm}^{-1}, \mathrm{~A}_{2}\right) \rightarrow \mid \mathrm{A}, 3 v_{2}+$ $\left.v_{4}\right\rangle\left(31782.2 \mathrm{~cm}^{-1}, \mathrm{~B}_{1}\right)$, and $\left|\mathrm{X}, 2 v_{2}+v_{3}+2 v_{6}\right\rangle\left(7362.3 \mathrm{~cm}^{-1}, \mathrm{~A}_{1}\right)$ $\rightarrow\left|\mathrm{A}, 4 v_{2}+v_{4}\right\rangle\left(32937.2 \mathrm{~cm}^{-1}, \mathrm{~B}_{1}\right)$ are in near-resonance with the
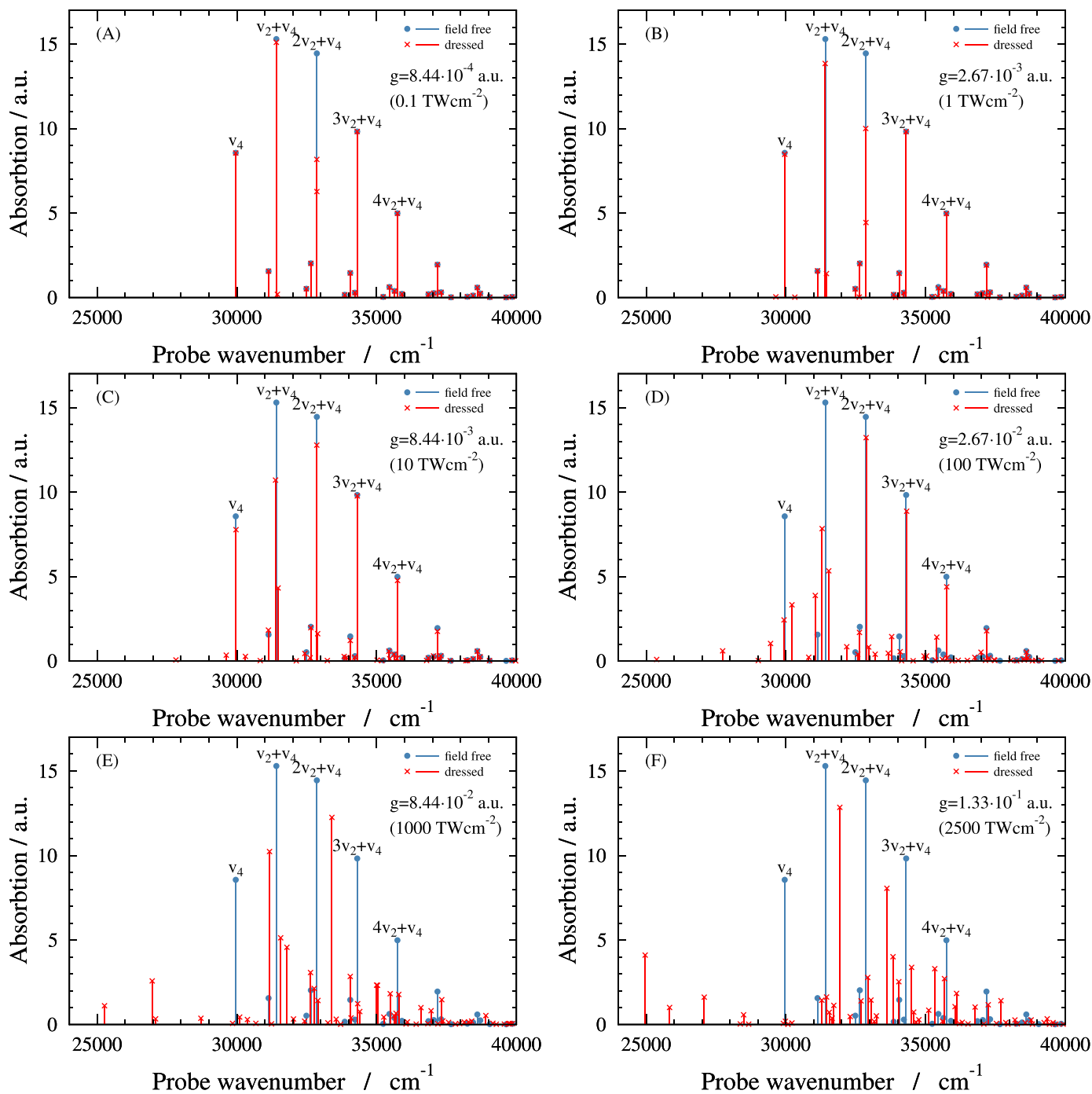

FIG. 7. Field-dressed (red) and field-free (blue) spectra of the $\mathrm{H}_{2} \mathrm{CO}$ molecule obtained with the $2 \mathrm{D}\left(v_{2}, v_{4}\right)$ quantum-dynamical model. The cavity wavenumber equals $\omega_{\mathrm{c}}=25385.2 \mathrm{~cm}^{-1}$, and the coupling strength values are indicated in panels $(\mathrm{A})-(\mathrm{F})$. Peaks of the field-free spectrum in the $v_{4}$ (out-of-plane bend) progression with different numbers of quanta in the $v_{2}(\mathrm{C}=0$ stretch $)$ vibrational mode are labeled $n v_{2}+v_{4}$, where $n=0, \ldots, 4$. 

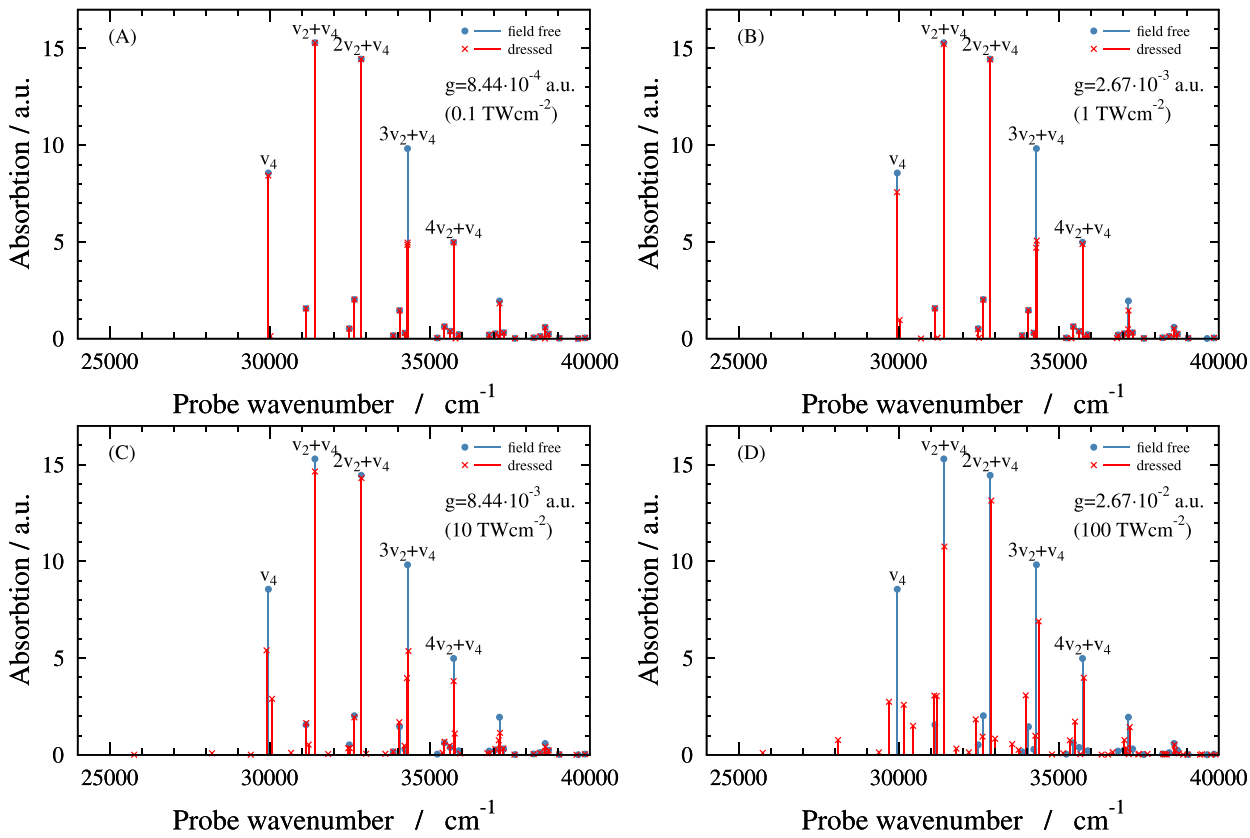

FIG. 8. Field-dressed (red) and field free (blue) spectra of the $\mathrm{H}_{2} \mathrm{CO}$ molecule obtained with the $2 \mathrm{D}\left(v_{2}, v_{4}\right)$ quantumdynamical model. The cavity wavenumber equals $\omega_{\mathrm{c}}=25764.8 \mathrm{~cm}^{-1}$, and the coupling strength values are indicated in panels $(A)-(F)$. Peaks of the field-free spectrum in the $v_{4}$ (out-of-plane bend) progression with different numbers of quanta in the $v_{2}(\mathrm{C}=\mathrm{O}$ stretch) vibrational mode are labeled $n v_{2}+v_{4}$, where $n=0, \ldots, 4$.
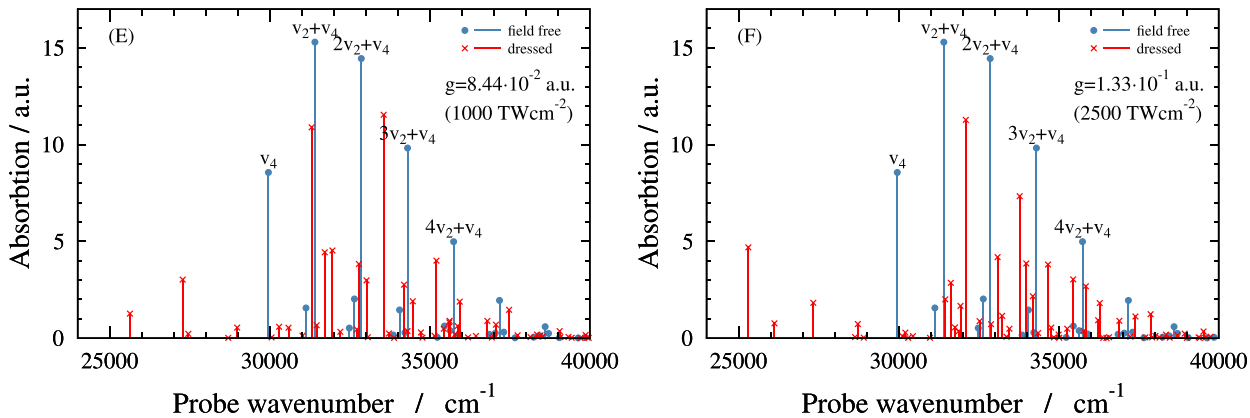

cavity wavenumber. As a consequence, dressed states with admixtures of $\mathrm{X}$ and A vibrational states are formed. An important difference from the dressed case of $\omega_{\mathrm{c}}=23794.0 \mathrm{~cm}^{-1}$ is that $\left|\mathrm{A}, 3 v_{2}+v_{4}\right\rangle$ can be coupled to $\left|\mathrm{X}, v_{3}+2 v_{4}+2 v_{6}\right\rangle$ by the $y$ TDM component and to $\left|\mathrm{X}, 3 v_{4}+v_{5}\right\rangle$ by the $x$ TDM component. Therefore, depending on the polarization, the field-free peak $3 v_{2}+v_{4}$ splits into three $\left(\mathbf{e}=\mathbf{e}_{x y}\right)$ or two peaks $\left(\mathbf{e}=\mathbf{e}_{y}\right.$ or $\left.\mathbf{e}=\mathbf{e}_{x}\right)$ in the dressed spectrum. As the states $\left|\mathrm{A}, 4 v_{2}+v_{4}\right\rangle$ and $\left|\mathrm{X}, 2 v_{2}+v_{3}+2 v_{6}\right\rangle$ can be coupled by the $y$ component of the TDM, the field-free peak $4 v_{2}+v_{4}$ splits into two peaks in the dressed case if $\mathbf{e}=\mathbf{e}_{x y}$ or $\mathbf{e}=\mathbf{e}_{y}$ and no splitting is observed if $\mathbf{e}=\mathbf{e}_{x}$. These results highlight the impact of the field polarization on the absorption spectra of molecules coupled to a cavity mode.

\section{B. Two-dimensional results}

In this subsection, results are presented for the $2 \mathrm{D}\left(v_{2}, v_{4}\right)$ model that treats only the $v_{2}$ and $v_{4}$ vibrational modes. The reason for choosing $v_{2}$ and $v_{4}$ as active vibrational modes is twofold. Displacements along the $v_{4}$ vibrational mode break the $C_{2} v$ symmetry of the $\mathrm{X}$ equilibrium structure and produce nonzero TDM, and the considerable difference in the $\mathrm{C}=\mathrm{O}$ equilibrium bond lengths of the electronic states $\mathrm{X}$ and $\mathrm{A}$ gives rise to several nonzero FranckCondon factors along the $v_{2}$ vibrational mode. Therefore, any reduced-dimensional description of $\mathrm{H}_{2} \mathrm{CO}$ should take into account the $v_{2}$ and $v_{4}$ vibrational modes.

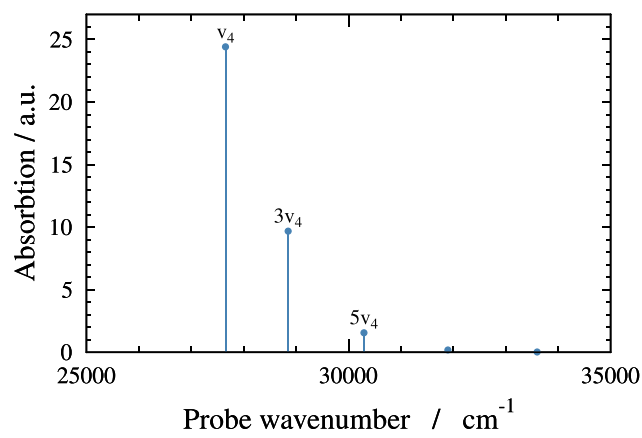

FIG. 9. Absorption spectrum of the field-free $\mathrm{H}_{2} \mathrm{CO}$ molecule obtained with the $1 \mathrm{D}\left(v_{4}\right)$ quantum-dynamical model. Peaks are labeled $n v_{4}$, where $n$ is the number of quanta in the $v_{4}$ (out-of-plane bend) vibrational mode. 
Figure 6 displays the field-free spectrum of $\mathrm{H}_{2} \mathrm{CO}$ obtained with the $2 \mathrm{D}\left(v_{2}, v_{4}\right)$ model. In Fig. 6, peaks that are members of the $v_{4}$ progression are explicitly labeled, similar to the $6 \mathrm{D}$ field-free spectrum in Fig. 3. Although the overall structures of the $2 \mathrm{D}\left(v_{2}, v_{4}\right)$ and $6 \mathrm{D}$ field-free spectra look similar, the $2 \mathrm{D}\left(v_{2}, v_{4}\right)$ model has several limitations: the remaining four modes $\left(v_{1}, v_{3}, v_{5}, v_{6}\right)$ are neglected, the $2 \mathrm{D}\left(v_{2}, v_{4}\right)$ eigenstates can be only of $\mathrm{A}_{1}$ or $\mathrm{B}_{1}$ symmetries, displacements along the $v_{2}$ and $v_{4}$ vibrational modes can produce TDM parallel to the body-fixed $y$ axis ( $x$ and $z$ components of the TDM are zero), and the PDM vector is always perpendicular to the TDM vector if the $2 \mathrm{D}\left(v_{2}, v_{4}\right)$ model is applied.

Given the limitations of the $2 \mathrm{D}\left(v_{2}, v_{4}\right)$ model, it is interesting to investigate to what extent the $2 \mathrm{D}\left(v_{2}, v_{4}\right)$ dressed spectra can reproduce the effects observed in the $6 \mathrm{D}$ dressed spectra. As only the vibrational modes $v_{2}$ and $v_{4}$ are considered, the $2 \mathrm{D}\left(v_{2}, v_{4}\right)$ model fails to provide sensible results if $\mathrm{X}$ and A vibrational states with excitations in modes other than $v_{2}$ and $v_{4}$ are coupled by the cavity mode (as is the case if the 6D model is used with $\omega_{\mathrm{c}}=25575.0 \mathrm{~cm}^{-1}$ ). Moreover, if the electric field is perpendicular to the body-fixed $y$ axis, no effects appear in the $2 \mathrm{D}\left(v_{2}, v_{4}\right)$ dressed spectrum as only the $y$ component of the TDM can be nonzero in the $2 \mathrm{D}\left(v_{2}, v_{4}\right)$ case. As we have seen in Subsection III A, in the 6D dressed case with $\omega_{\mathrm{c}}=23794.0 \mathrm{~cm}^{-1}$, splittings of the field-free peaks $2 v_{2}+v_{4}$ and $3 v_{2}+v_{4}$ can be attributed to interactions of the states $\left|\mathrm{X}, 6 v_{4}\right\rangle$ and $\left|\mathrm{A}, 2 v_{2}+v_{4}\right\rangle$ and $\left|\mathrm{X}, 2 v_{2}+4 v_{4}\right\rangle$ and $\left|\mathrm{A}, 3 v_{2}+v_{4}\right\rangle$, all of which can be obtained by the $2 \mathrm{D}\left(v_{2}, v_{4}\right)$ model. In the following, we shall focus
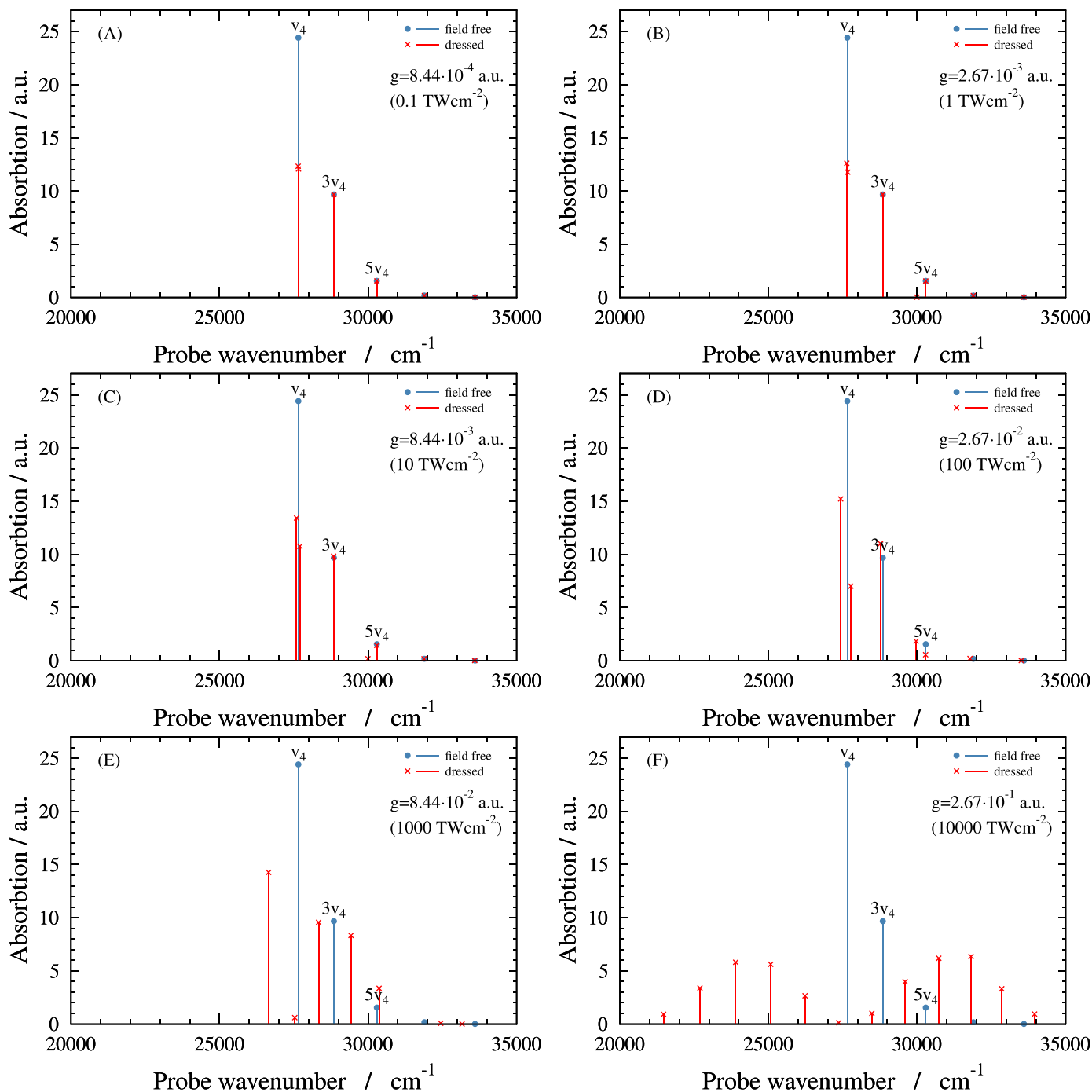

FIG. 10. Field-dressed (red) and field-free (blue) spectra of the $\mathrm{H}_{2} \mathrm{CO}$ molecule obtained with the $1 \mathrm{D}\left(v_{4}\right)$ quantum-dynamical model. The cavity wavenumber equals $\omega_{\mathrm{c}}=27653.3 \mathrm{~cm}^{-1}$, and the coupling strength values are indicated in panels $(\mathrm{A})-(\mathrm{F})$. Peaks of the field-free spectrum are labeled $n v_{4}$, where $n$ is the number of quanta in the $v_{4}$ (out-of-plane bend) vibrational mode. 
on the effects produced by the field-induced interactions of the state pairs $\left|\mathrm{X}, 6 v_{4}\right\rangle-\left|\mathrm{A}, 2 v_{2}+v_{4}\right\rangle$ and $\left|\mathrm{X}, 2 v_{2}+4 v_{4}\right\rangle-\left|\mathrm{A}, 3 v_{2}+v_{4}\right\rangle$ in the $2 \mathrm{D}\left(v_{2}, v_{4}\right)$ dressed spectrum.

Figures 7 and 8 display $2 \mathrm{D}\left(v_{2}, v_{4}\right)$ dressed spectra with $\omega_{\mathrm{c}}=25385.2 \mathrm{~cm}^{-1}$ (resonant with the transition $\left|\mathrm{X}, 6 v_{4}\right\rangle$ $\left.\rightarrow\left|\mathrm{A}, 2 v_{2}+v_{4}\right\rangle\right)$ and $\omega_{\mathrm{c}}=25764.8 \mathrm{~cm}^{-1}$ (resonant with the transition $\left.\left|\mathrm{X}, 2 v_{2}+4 v_{4}\right\rangle \rightarrow\left|\mathrm{A}, 3 v_{2}+v_{4}\right\rangle\right)$ for different coupling strength values with $\mathbf{e}=(0,1,0)$. For the lowest coupling strength value of $g=8.44$ $\times 10^{-4}$ a.u., only the field-free peaks $2 v_{2}+v_{4}\left(\omega_{\mathrm{c}}=25385.2 \mathrm{~cm}^{-1}\right.$, Fig. 7) and $3 v_{2}+v_{4}\left(\omega_{\mathrm{c}}=25764.8 \mathrm{~cm}^{-1}\right.$, Fig. 8) split into two peaks in the $2 \mathrm{D}\left(v_{2}, v_{4}\right)$ dressed spectrum. In contrast to the $6 \mathrm{D}$ dressed spectrum with $\omega_{\mathrm{c}}=23794.0 \mathrm{~cm}^{-1}$ and $\mathbf{e}=(0,1,0)$, no simultaneous splittings of $2 v_{2}+v_{4}$ and $3 v_{2}+v_{4}$ are visible in any of the $2 \mathrm{D}\left(v_{2}, v_{4}\right)$ dressed spectra with $g=8.44 \times 10^{-4}$ a.u.

As $g$ increases, the $2 \mathrm{D}\left(v_{2}, v_{4}\right)$ dressed spectra gradually diverge from the $2 \mathrm{D}\left(v_{2}, v_{4}\right)$ field-free spectrum. Besides the simultaneous splittings of multiple field-free peaks, the $2 \mathrm{D}\left(v_{2}, v_{4}\right)$ dressed spectra show the emergence of additional peaks, which can be attributed to intensity borrowing induced by the quantum LICI. Finally, for the highest $g$ values, separation of two peak groups can be observed in both Figs. 7 and 8 . This is due to the increasing separation of the lower and upper polaritonic PESs.

\section{One-dimensional results}

Finally, we investigate a one-dimensional quantum-dynamical model, called the $1 \mathrm{D}\left(v_{4}\right)$ model, treating only the $v_{4}$ vibrational mode of $\mathrm{H}_{2} \mathrm{CO}$. Figure 9 shows the $1 \mathrm{D}\left(v_{4}\right)$ field-free spectrum that resembles neither the $6 \mathrm{D}$ nor the $2 \mathrm{D}\left(v_{2}, v_{4}\right)$ field-free spectra. Not surprisingly, as the $v_{2}$ vibrational mode is not taken into account by the $1 \mathrm{D}\left(v_{4}\right)$ model, the $v_{4}$ and $3 v_{4}$ progressions present in the higherdimensional spectra reduce to single peaks in the $1 \mathrm{D}\left(v_{4}\right)$ field-free spectrum, as indicated by the labels $n v_{4}$ in Fig. 9.

1D $\left(v_{4}\right)$ dressed spectra are shown in Fig. 10 with a cavity wavenumber of $\omega_{c}=27653.3 \mathrm{~cm}^{-1}$ for a broad range of coupling strength values. This wavenumber value resonantly couples $|\mathrm{X}, 0\rangle$ (X vibrational ground state) to $\left|\mathrm{A}, v_{4}\right\rangle$, and the $v_{4}$ peak of the $1 \mathrm{D}\left(v_{4}\right)$ is split in the dressed case as a consequence. At the lowest coupling strength value of $g=8.44 \times 10^{-4}$ a.u., the $v_{4}$ peak splits into two lines that correspond to spectral transitions to two dressed states that can be approximately described as $\left(|\mathrm{X}, 0\rangle|1\rangle \pm\left|\mathrm{A}, v_{4}\right\rangle|0\rangle\right) / \sqrt{2}$. At higher $g$ values, the structure of the dressed spectrum gradually changes, and finally, for the highest $g$ value applied, one can again notice the separation of two peak groups due to the increasing separation of the lower and upper polaritonic PESs.

\section{CONCLUSIONS}

In this work, the cavity-dressed absorption spectrum of the $\mathrm{H}_{2} \mathrm{CO}$ (formaldehyde) molecule has been investigated with a fulldimensional $(6 \mathrm{D})$ and two reduced-dimensional $\left[2 \mathrm{D}\left(v_{2}, v_{4}\right)\right.$ and $1 \mathrm{D}\left(v_{4}\right)$ ] quantum-dynamical models. Numerical simulations were performed with different cavity frequencies for a wide range of the cavity coupling strength parameter. We chose this system as it does not possess any natural nonadiabatic phenomena in the studied region of the nuclear configuration space. Consequently, neither natural conical intersections nor natural avoided crossings occur, and only quantum-light-induced nonadiabatic effects can be present in the system.

Accurate 6D quantum-dynamical calculations undoubtedly show the impact of the quantum LICI on the high-energy region of the vibronic spectrum of $\mathrm{H}_{2} \mathrm{CO}$ even for the relatively weak cavity coupling strength value employed. Noticeable splittings appear in the dressed spectra compared to the field-free case. For the $2 \mathrm{D}\left(v_{2}\right.$, $\left.v_{4}\right)$ and $1 \mathrm{D}\left(v_{4}\right)$ models we have obtained dressed spectra for coupling strength values higher than that used in the all-mode calculations. For both reduced-dimensional models, one finds that the dressed spectra gradually diverge from the field-free spectrum as the cavity coupling strength $g$ increases. For the highest $g$ values, a separation of two peak groups can be observed in the dressed spectra due to the increasing separation of the lower and upper polaritonic PESs. Both dressed and field-free spectra obtained with the $1 \mathrm{D}\left(v_{4}\right)$ and $2 \mathrm{D}\left(v_{2}, v_{4}\right)$ models differ significantly. Comparison of the $1 \mathrm{D}\left(v_{4}\right)$ and $2 \mathrm{D}\left(v_{2}, v_{4}\right)$ spectra to the $6 \mathrm{D}$ spectrum clearly shows that at least a $2 \mathrm{D}$ approximation is needed to even crudely describe the absorption spectrum of $\mathrm{H}_{2} \mathrm{CO}$ coupled to a cavity mode. For the $2 \mathrm{D}\left(v_{2}, v_{4}\right)$ dressed spectra, not only the simultaneous splittings of multiple field-free peaks can be observed but also additional peaks appear, which can be seen as a clear fingerprint of the intensity borrowing effect induced by the quantum LICI.

It becomes clear from the present first full-dimensional investigation of a polyatomic molecule in a cavity that reduceddimensional models have limitations and should be considered with care. On the other hand, such models are useful and may be even indispensable when investigating strong coupling to the cavity, where currently accurate full-dimensional calculations are still difficult to perform.

\section{ACKNOWLEDGMENTS}

The authors gratefully acknowledge Professor Joel Bowman for providing Fortran subroutines for the $S_{0}$ and $S_{1}$ potential energy surfaces. We are indebted to Gerrit Groenhof for fruitful discussions. This research was supported by the EU-funded Hungarian Grant (Award No. EFOP-3.6.2-16-2017-00005). The authors are grateful to NKFIH for financial support (Grant Nos. K128396 and PD124699).

\section{DATA AVAILABILITY}

The data that support the findings of this study are available from the corresponding author upon reasonable request.

\section{REFERENCES}

${ }^{1}$ H. Köppel, W. Domcke, and L. S. Cederbaum, Adv. Chem. Phys. 57, 59 (1984)

${ }^{2}$ D. R. Yarkony, Rev. Mod. Phys. 68, 985 (1996).

${ }^{3}$ M. Baer, Phys. Rep. 358, 75 (2002).

${ }^{4}$ G. A. Worth and L. S. Cederbaum, Ann. Rev. Phys. Chem. 55, 127 (2004).

${ }^{5}$ W. Domcke, D. R. Yarkony, and H. Köppel, Conical Intersections (World Scientific, 2004).

${ }^{6} \mathrm{M}$. Baer, Beyond Born-Oppenheimer: Electronic Non-Adiabatic Coupling Terms and Conical Intersections (Wiley, New York, 2006).

${ }^{7}$ F. Bernardi, M. Olivucci, and M. A. Robb, Chem. Soc. Rev. 25, 321 (1996).

${ }^{8}$ G. Groenhof, L. V. Schäfer, M. Boggio-Pasqua, M. Goette, H. Grubmüller, and M. A. Robb, J. Am. Chem. Soc. 129, 6812 (2007) 
${ }^{9}$ M. Boggio-Pasqua, G. Groenhof, L. V. Schäfer, H. Grubmüller, and M. A. Robb, J. Am. Chem. Soc. 129, 10996 (2007).

${ }^{10}$ A. J. Musser, M. Liebel, C. Schnedermann, T. Wende, T. B. Kehoe, A. Rao, and P. Kukura, Nat. Phys. 11, 352 (2015).

${ }^{11}$ A. von Conta, A. Tehlar, A. Schletter, Y. Arasaki, K. Takatsuka, and H. J. Wörner, Nat. Commun. 9, 3162 (2018).

${ }^{12}$ M. E. Corrales, J. González-Vázquez, R. de Nalda, and L. Bañares, J. Phys. Chem. Lett. 10, 138 (2018).

${ }^{13}$ T. J. Martinez, Nature 467, 412 (2010).

${ }^{14}$ C. Xie, J. Ma, X. Zhu, D. R. Yarkony, D. Xie, and H. Guo, J. Am. Chem. Soc. 138, 7828 (2016).

${ }^{15}$ J. A. DeVine, M. L. Weichman, X. Zhou, J. Ma, B. Jiang, H. Guo, and D. M. Neumark, J. Am. Chem. Soc. 138, 16417 (2016).

${ }^{16}$ K. C. Woo, D. H. Kang, and S. K. Kim, J. Am. Chem. Soc. 139, 17152 (2017).

${ }^{17}$ B. F. E. Curchod and T. J. Martínez, Chem. Rev. 118, 3305 (2018).

${ }^{18}$ I. G. Ryabinkin, L. Joubert-Doriol, and A. F. Izmaylov, Acc. Chem. Res. 50, 1785 (2017).

${ }^{19}$ C. Xie, C. L. Malbon, H. Guo, and D. R. Yarkony, Acc. Chem. Res. 52, 501 (2019).

${ }^{20}$ N. Moiseyev, M. Šindelka, and L. S. Cederbaum, J. Phys. B: At., Mol. Opt. Phys. 41, 221001 (2008).

${ }^{21}$ G. J. Halász, Á. Vibók, and L. S. Cederbaum, J. Phys. Chem. Lett. 6, 348 (2015).

${ }^{22}$ M. Kowalewski, K. Bennett, and S. Mukamel, J. Phys. Chem. Lett. 7, 2050 (2016).

${ }^{23}$ A. Csehi, G. J. Halász, L. S. Cederbaum, and Á. Vibók, J. Phys. Chem. Lett. 8, 1624 (2017).

${ }^{24}$ G. J. Halász, Á. Vibók, M. Šindelka, N. Moiseyev, and L. S. Cederbaum, J. Phys. B: At., Mol. Opt. Phys. 44, 175102 (2011).

${ }^{25}$ G. J. Halász, M. Šindelka, N. Moiseyev, L. S. Cederbaum, and Á. Vibók, J. Phys. Chem. A 116, 2636 (2011).

${ }^{26}$ A. Csehi, G. J. Halász, L. S. Cederbaum, and Á. Vibók, Faraday Discuss. 194, 479 (2016).

${ }^{27}$ T. Szidarovszky, G. J. Halász, A. G. Császár, L. S. Cederbaum, and Á. Vibók, J. Phys. Chem. Lett. 9, 2739 (2018).

${ }^{28}$ P. V. Demekhin and L. S. Cederbaum, J. Chem. Phys. 139, 154314 (2013).

${ }^{29}$ J. Kim, H. Tao, J. L. White, V. S. Petrović, T. J. Martinez, and P. H. Bucksbaum, J. Phys. Chem. A 116, 2758 (2012).

${ }^{30}$ C. Fábri, B. Lasorne, G. J. Halász, L. S. Cederbaum, and Á. Vibók, J. Phys. Chem. Lett. 11, 5324 (2020).

${ }^{31}$ A. Natan, M. R. Ware, V. S. Prabhudesai, U. Lev, B. D. Bruner, O. Heber, and P. H. Bucksbaum, Phys. Rev. Lett. 116, 143004 (2016).

${ }^{32}$ M. E. Corrales, J. González-Vázquez, G. Balerdi, I. R. Solá, R. de Nalda, and L. Bañares, Nat. Chem. 6, 785 (2014).

${ }^{33}$ M. Kübel, M. Spanner, Z. Dube, A. Y. Naumov, S. Chelkowski, A. D. Bandrauk, M. J. Vrakking, P. B. Corkum, D. Villeneuve, and A. Staudte, Nat. Commun. 11, 2596 (2020)

${ }^{34}$ J. A. Hutchison, T. Schwartz, C. Genet, E. Devaux, and T. W. Ebbesen, Angew. Chem., Int. Ed. 51, 1592 (2012).

${ }^{35}$ T. W. Ebbesen, Acc. Chem. Res. 49, 2403 (2016).

${ }^{36}$ R. Chikkaraddy, B. de Nijs, F. Benz, S. J. Barrow, O. A. Scherman, E. Rosta, A. Demetriadou, P. Fox, O. Hess, and J. J. Baumberg, Nature 535, 127 (2016).

${ }^{37}$ X. Zhong, T. Chervy, S. Wang, J. George, A. Thomas, J. A. Hutchison, E. Devaux, C. Genet, and T. W. Ebbesen, Angew. Chem. 128, 6310 (2016).

${ }^{38}$ J. Galego, F. J. Garcia-Vidal, and J. Feist, Phys. Rev. X 5, 041022 (2015).

${ }^{39}$ J. Feist, J. Galego, and F. J. Garcia-Vidal, ACS Photonics 5, 205 (2017).

${ }^{40}$ H. L. Luk, J. Feist, J. J. Toppari, and G. Groenhof, J. Chem. Theory Comput. 13, 4324 (2017).

${ }^{41}$ J. Flick, M. Ruggenthaler, H. Appel, and A. Rubio, Proc. Natl. Acad. Sci. U. S. A. 114, 3026 (2017).

${ }^{42}$ J. Flick, H. Appel, M. Ruggenthaler, and A. Rubio, J. Chem. Theory Comput. 13, 1616 (2017).

${ }^{43}$ T. Szidarovszky, G. J. Halász, A. G. Császár, L. S. Cederbaum, and Á. Vibók, J. Phys. Chem. Lett. 9, 6215 (2018).
${ }^{44}$ R. F. Ribeiro, L. A. Martínez-Martínez, M. Du, J. Campos-Gonzalez-Angulo, and J. Yuen-Zhou, Chem. Sci. 9, 6325 (2018).

${ }^{45}$ J. Flick, N. Rivera, and P. Narang, Nanophotonics 7, 1479 (2018).

${ }^{46}$ A. Csehi, M. Kowalewski, G. J. Halász, and Á. Vibók, New J. Phys. 21, 093040 (2019).

${ }^{47}$ G. Groenhof and J. J. Toppari, J. Phys. Chem. Lett. 9, 4848 (2018).

${ }^{48}$ O. Vendrell, Phys. Rev. Lett. 121, 253001 (2018).

${ }^{49}$ J. F. Triana, D. Peláez, and J. L. Sanz-Vicario, J. Phys. Chem. A 122, 2266 (2018).

${ }^{50}$ J. B. Pérez-Sánchez and J. Yuen-Zhou, J. Phys. Chem. Lett. 11, 152 (2019).

${ }^{51}$ A. Mandal and P. Huo, J. Phys. Chem. Lett. 10, 5519 (2019).

${ }^{52}$ I. S. Ulusoy, J. A. Gomez, and O. Vendrell, J. Phys. Chem. A 123, 8832 (2019).

${ }^{53}$ M. Litinskaya and F. Herrera, Phys. Rev. B 99, 041107 (2019).

${ }^{54}$ J. F. Triana and J. L. Sanz-Vicario, Phys. Rev. Lett. 122, 063603 (2019).

${ }^{55}$ F. Herrera, Chem 6, 7 (2020).

${ }^{56}$ E. Davidsson and M. Kowalewski, J. Phys. Chem. A 124, 4672 (2020).

${ }^{57}$ B. Gu and S. Mukamel, Chem. Sci. 11, 1290 (2020).

${ }^{58}$ A. Mandal, S. Montillo Vega, and P. Huo, J. Phys. Chem. Lett. 11, 9215 (2020).

${ }^{59}$ J. d. Pino, J. Feist, and F. J. Garcia-Vidal, New J. Phys. 17, 053040 (2015).

${ }^{60}$ A. D. Dunkelberger, B. T. Spann, K. P. Fears, B. S. Simpkins, and J. C. Owrutsky, Nat. Commun. 7, 13504 (2016).

${ }^{61}$ F. Herrera and F. C. Spano, ACS Photonics 5, 65 (2017).

${ }^{62}$ W. Ahn, I. Vurgaftman, A. D. Dunkelberger, J. C. Owrutsky, and B. S. Simpkins, ACS Photonics 5, 158 (2017).

${ }^{63}$ A. Thomas, J. George, A. Shalabney, M. Dryzhakov, S. J. Varma, J. Moran, T. Chervy, X. Zhong, E. Devaux, C. Genet, J. A. Hutchison, and T. W. Ebbesen, Angew. Chem., Int. Ed. 55, 11462 (2016).

${ }^{64}$ R. M. A. Vergauwe, J. George, T. Chervy, J. A. Hutchison, A. Shalabney, V. Y. Torbeev, and T. W. Ebbesen, J. Phys. Chem. Lett. 7, 4159 (2016).

${ }^{65}$ T. Chervy, A. Thomas, E. Akiki, R. M. A. Vergauwe, A. Shalabney, J. George, E. Devaux, J. A. Hutchison, C. Genet, and T. W. Ebbesen, ACS Photonics 5, 217 (2017).

${ }^{66}$ R. M. A. Vergauwe, A. Thomas, K. Nagarajan, A. Shalabney, J. George, T. Chervy, M. Seidel, E. Devaux, V. Torbeev, and T. W. Ebbesen, Angew. Chem., Int. Ed. 58, 15324 (2019).

${ }^{67}$ A. Thomas, L. Lethuillier-Karl, K. Nagarajan, R. M. A. Vergauwe, J. George, T. Chervy, A. Shalabney, E. Devaux, C. Genet, J. Moran, and T. W. Ebbesen, Science 363, 615 (2019).

${ }^{68}$ J. P. Long and B. S. Simpkins, ACS Photonics 2, 130 (2014).

${ }^{69}$ M. Muallem, A. Palatnik, G. D. Nessim, and Y. R. Tischler, J. Phys. Chem. Lett. 7, 2002 (2016).

${ }^{70}$ R. Damari, O. Weinberg, D. Krotkov, N. Demina, K. Akulov, A. Golombek, T. Schwartz, and S. Fleischer, Nat. Commun. 10, 3248 (2019).

${ }^{71}$ J. A. Campos-Gonzalez-Angulo, R. F. Ribeiro, and J. Yuen-Zhou, Nat. Commun. 10, 4685 (2019).

${ }^{72}$ S. Kéna-Cohen and J. Yuen-Zhou, ACS Cent. Sci. 5, 386 (2019).

${ }^{73}$ J. Galego, C. Climent, F. J. Garcia-Vidal, and J. Feist, Phys. Rev. X 9, 021057 (2019).

${ }^{74}$ J. A. Campos-Gonzalez-Angulo and J. Yuen-Zhou, J. Chem. Phys. 152, 161101 (2020).

${ }^{75}$ X. Li, A. Mandal, and P. Huo, chemRxiv:12915701.v1 (2020).

${ }^{76}$ A. Mandal, T. D. Krauss, and P. Huo, J. Phys. Chem. B 124, 6321 (2020).

${ }^{77}$ C. Fábri, G. J. Halász, L. S. Cederbaum, and Á. Vibók, "Born-Oppenheimer approximation in optical cavities: From success to breakdown," Chem. Sci. (published online) (2020).

${ }^{78}$ M. Araujo, B. Lasorne, M. J. Bearpark, and M. A. Robb, J. Phys. Chem. A 112, 7489 (2008).

${ }^{79}$ M. Araújo, B. Lasorne, A. L. Magalhães, G. A. Worth, M. J. Bearpark, and M. A. Robb, J. Chem. Phys. 131, 144301 (2009).

${ }^{80}$ M. Araújo, B. Lasorne, A. L. Magalhães, M. J. Bearpark, and M. A. Robb, J. Phys. Chem. A 114, 12016 (2010).

${ }^{81}$ A. Raab, G. A. Worth, H.-D. Meyer, and L. S. Cederbaum, J. Chem. Phys. 110, 936 (1999). 
${ }^{82}$ C. Cattarius, G. A. Worth, H.-D. Meyer, and L. S. Cederbaum, J. Chem. Phys. 115, 2088 (2001).

${ }^{83}$ C. Cohen-Tannoudji, J. Dupont-Roc, and G. Grynberg, Atom-Photon Interactions: Basic Processes and Applications (Wiley-VCH Verlag GmbH and Co. KGaA, Weinheim, 2004).

${ }^{84}$ R. S. Mulliken, J. Chem. Phys. 23, 1997 (1955).

${ }^{85}$ X. Wang, P. L. Houston, and J. M. Bowman, Philos. Trans. R. Soc., A 375, 20160194 (2017).

${ }^{86}$ B. Fu, B. C. Shepler, and J. M. Bowman, J. Am. Chem. Soc. 133, 7957 (2011).

${ }^{87}$ E. Mátyus, G. Czakó, and A. G. Császár, J. Chem. Phys. 130, 134112 (2009).
${ }^{88}$ C. Fábri, E. Mátyus, and A. G. Császár, J. Chem. Phys. 134, 074105 (2011).

${ }^{89}$ A. G. Császár, C. Fábri, T. Szidarovszky, E. Mátyus, T. Furtenbacher, and G. Czakó, Phys. Chem. Chem. Phys. 14, 1085 (2012).

${ }^{90}$ X. Chapuisat and C. Iung, Phys. Rev. A 45, 6217 (1992).

${ }^{91}$ C. Eckart, Phys. Rev. 47, 552 (1935).

${ }^{92} \mathrm{P}$. Bunker and P. Jensen, Molecular Symmetry and Spectroscopy (NRC Research Press, 2006).

${ }^{93}$ C. Fábri, M. Quack, and A. G. Császár, J. Chem. Phys. 147, 134101 (2017).

${ }^{94}$ D. J. Clouthier and D. A. Ramsay, Ann. Rev. Phys. Chem. 34, 31 (1983).

${ }^{95}$ M. Bonfanti, J. Petersen, P. Eisenbrandt, I. Burghardt, and E. Pollak, J. Chem. Theory Comput. 14, 5310 (2018). 